\title{
LA LOGIQUE DU DON DANS LA TRANSMISSION DES ENTREPRISES FAMILIALES \\ Delphine Lobet
}

\author{
Presses de Sciences Po (P.F.N.S.P.) | «Sociétés contemporaines »
}

2006/2 n ${ }^{0} 62 \mid$ pages 27 à 47

ISSN 1150-1944

ISBN 2724630599

Article disponible en ligne à l'adresse :

https://www.cairn.info/revue-societes-contemporaines-2006-2-page-27.htm

\section{Pour citer cet article :}

Delphine Lobet, « La logique du don dans la transmission des entreprises familiales », Sociétés contemporaines 2006/2 (nº 62), p. 27-47.

DOI 10.3917/soco.062.0027

Distribution électronique Cairn.info pour Presses de Sciences Po (P.F.N.S.P.).

(C) Presses de Sciences Po (P.F.N.S.P.). Tous droits réservés pour tous pays.

La reproduction ou représentation de cet article, notamment par photocopie, n'est autorisée que dans les limites des conditions générales d'utilisation du site ou, le cas échéant, des conditions générales de la licence souscrite par votre établissement. Toute autre reproduction ou représentation, en tout ou partie, sous quelque forme et de quelque manière que ce soit, est interdite sauf accord préalable et écrit de l'éditeur, en dehors des cas prévus par la législation en vigueur en France. Il est précisé que son stockage dans une base de données est également interdit. 


\title{
LA LOGIQUE DU DON \\ DANS LA TRANSMISSION DES ENTREPRISES FAMILIALES
}

\begin{abstract}
RÉsUMí : La logique du don, telle que Mauss l'a théorisée dans son célèbre essai, semble particulièrement à même de rendre compte de la logique qui sous-tend la transmission des entreprises familiales. Tout comme le don exige d'être rendu, l'héritage exigerait d'être retransmis: transmettant à ses enfants, on rendrait à ses parents. Une enquête auprès des membres de cinq entreprises familiales wallonnes, représentant jusqu'à quatre générations, nous invite cependant à considérablement nuancer le système d'obligations du don. Contexte économique mais aussi tensions relationnelles, incompatibilité de l'entreprise avec d'autres projets, désenchantement du statut, ouverture et autonomisation des devenirs hors de la famille, font que, dans les faits, le cycle intergénérationnel a ses ratés.
\end{abstract}

\section{INTRODUCTION}

Lorsque l'on évoque les entreprises familiales et leurs transmissions, une série d'images viennent à l'esprit, qui mêlent famille, patrimoine et dynastie. Ces images s'imposent avec d'autant plus de force que sont longues les chaînes généalogiques maillées autour de l'entreprise. Il est une mythologie de l'héritage, du rapport aux ancêtres, selon laquelle l'histoire longue soumet ses sujets. En vertu de cette «piété laïque » que suscite l'héritage (Gotman, 1988 : 222), les chefs d'entreprise qui ont hérité de leur entreprise doivent être mus par l'obligation morale de la conserver, d'en prendre soin et de la faire passer aux générations suivantes. Il est en effet admis que « les biens hérités ne sont pas mis à la disposition personnelle des héritiers » et que « la transmission est pour une large part re-transmission » (Ibid. : 206, 157).

Comment comprendre la «piété laïque » et les devoirs associés aux héritages ? Anne Gotman a montré que les biens transmis « comme les objets donnés [ont] encore quelque chose de leur donateur » (Ibid. : 206). On retrouve là la théorie maussienne d'un don qui triangule les relations entre les êtres : le bien oblige et lie les personnes, ce qui est donné doit être reçu, pris et puis rendu. Transmettre à son tour, c'est donc répondre à l'injonction de rendre que formule le donné. L'héritage, anthume ou posthume, serait comme un don au cycle d'obligations linéarisé, un don dont le contre-don se réaliserait verticalement : on rendrait à ses ascendants en donnant à ses descendants (Gotman 1988 ; Godbout et Caillé, 1992). 
Mais Anne Gotman note également que « la retransmission, bien qu'idéale, est toutefois conditionnée par les circonstances de la vie de famille et plus radicalement par l'économie de rareté à laquelle la parentèle est elle aussi soumise » (Gotman, 1988 : 221). Les obligations du don ne seraient donc pas assez puissantes pour soumettre toutes les autres. Cette remarque nous pousse à nous demander quelles sont aujourd'hui les «circonstance familiales et économiques » qui mettraient en échec, ou du moins nuanceraient considérablement, la logique du don. Car, en effet, quand ils n'ont pas déjà vendu leur affaire, les héritiers-chefs d'entreprise interrogés dans le cadre de cette recherche se montrent plutôt circonspects quant à l'idée de la transmettre à leur tour. Mais cela ne signifie pas pour autant que ce dont ils ont hérité n'est pas un tant soit peu «spécial » pour eux ; il nous semble impossible d'abandonner cette idée qu'il y a bien un « esprit », « quelque chose du donateur » qui circule avec les biens transmis.

Aussi, prenons la mythologie au sérieux, faisons «comme si » la logique du don présidait aux échanges intergénérationnels et voyons ce qui conditionne la transformation de l'héritier (du donataire) en transmetteur (en donateur). Voyons d'où vient qu'ayant hérité d'une entreprise, l'on ne désire pas la transmettre ou que l'on reste plutôt passif face à cette possibilité. Par quoi l'enrayement de la logique du don peut-il s'expliquer?

\section{CINQ ENTREPRISES FAMILIALES}

Cette réflexion s'inscrit dans une recherche portant sur la transmission des entreprises familiales, c'est-à-dire sur la transmission d' « organisations de production de biens ou de services, à caractère commercial, dont la propriété et le pouvoir de gestion sont sous le contrôle effectif des membres d'une même famille » (d'après Hugron, 1998). Cette confusion de la propriété et de la gestion est reconnue caractériser plus souvent les petites structures que les grosses, c'est pourquoi on a choisi de ne s'intéresser qu'à des petites et moyennes entreprises de moins de cinquante travailleurs. Loin de composer la marge du tissu économique, ces entreprises représentent en Belgique 96,7\% des établissements privés et occupent près de $49 \%$ des travailleurs du secteur privé. La France connaît des chiffres comparables puisque $99 \%$ des entreprises privées sont des PME de moins de cinquante travailleurs. Bien sûr, toutes ces PME ne sont pas des entreprises familiales au sens où nous l'entendons, mais, en l'absence d'études qui les auraient dénombrées, on peut penser qu'il s'agit d'une bonne approximation. Michel Bauer notait d'ailleurs que, d'une part, « dans ces petites entreprises, le pouvoir est presque toujours concentré dans les mains d'un seul homme » et que, d'autre part, « les patrons de PME sont presque toujours personnellement propriétaires de leur entreprise » (1992: 151). Tout porte donc à croire que les entreprises familiales, typiques du «premier esprit du capitalisme» décrit par Boltanski et Chiapello (1999 : 57), restent à l'ère du « troisième esprit » très présentes dans nos économies.

Les résultats présentés ici se rapportent à l'étude de cinq entreprises wallonnes (Belgique) qui offrent un bon aperçu des figures typiques de l'entreprise familiale et des devenirs qui lui sont possibles. En effet, au moment de l'enquête (2003-2004), leur longévité variait de deux à cinq générations ; elles étaient dirigées par des fratries, des cousins, des couples ou une personne isolée ; trois d'entre elles avaient été 
récemment transmises au sein de la famille (direction et propriété) et les deux autres avaient été tout aussi récemment cédées à de grands groupes.

Les arbres ci-dessous présentent succinctement ces entreprises (activité, historique, taille), ils donnent un aperçu de leur profondeur généalogique et des relations des membres de la famille avec l'entreprise. Tous les noms sont fictifs, ainsi que les dates, qui ont été légèrement modifiées. Pour faciliter la lecture, nous avons emprunté à Daniel Bertaux et Isabelle Bertaux-Wiame (1988) leur système de distribution des prénoms : les prénoms des membres de la génération fondatrice commencent toujours par la lettre « $\mathrm{A} »$, ceux de la deuxième par « $\mathrm{B} »$, etc.

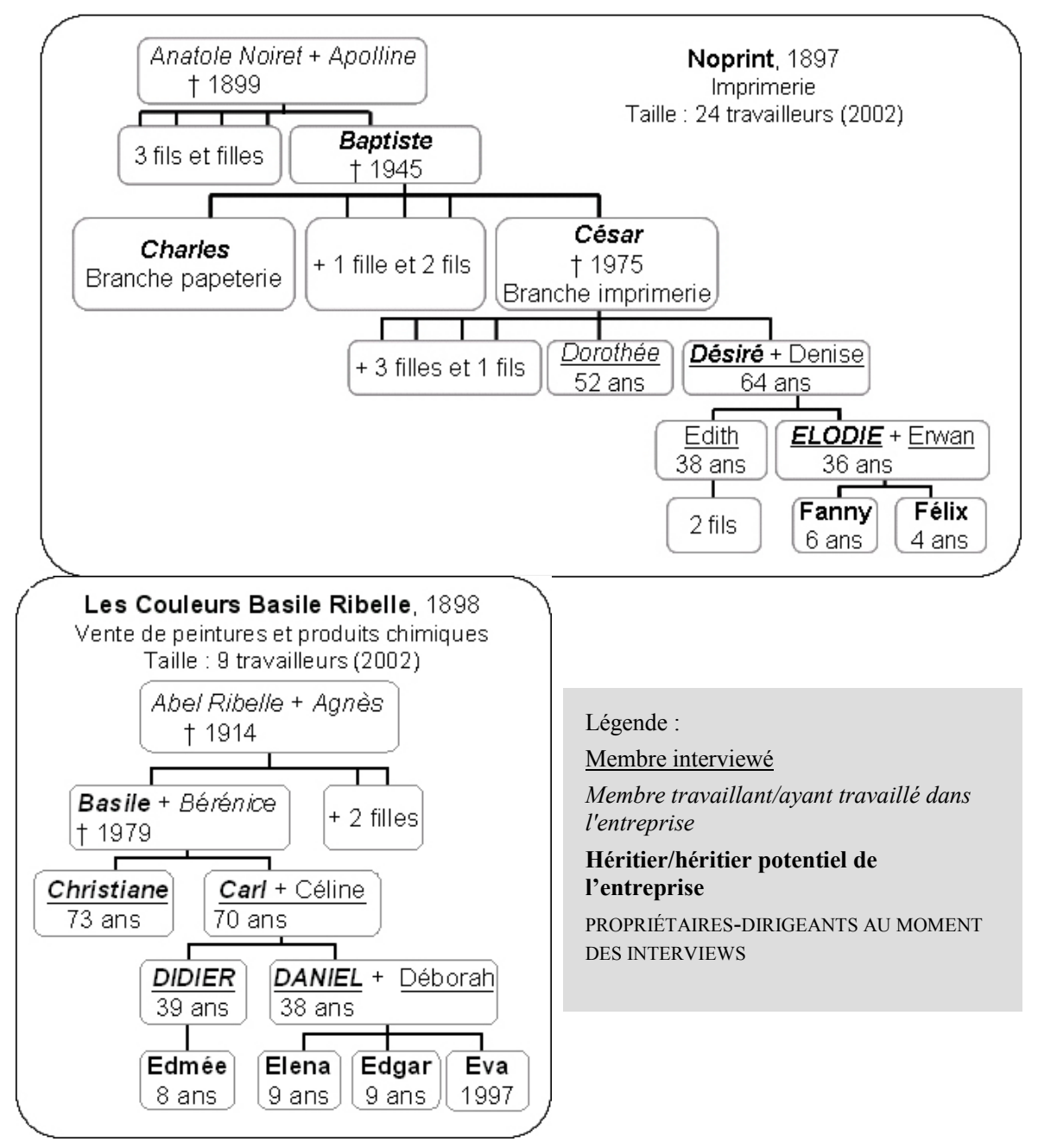



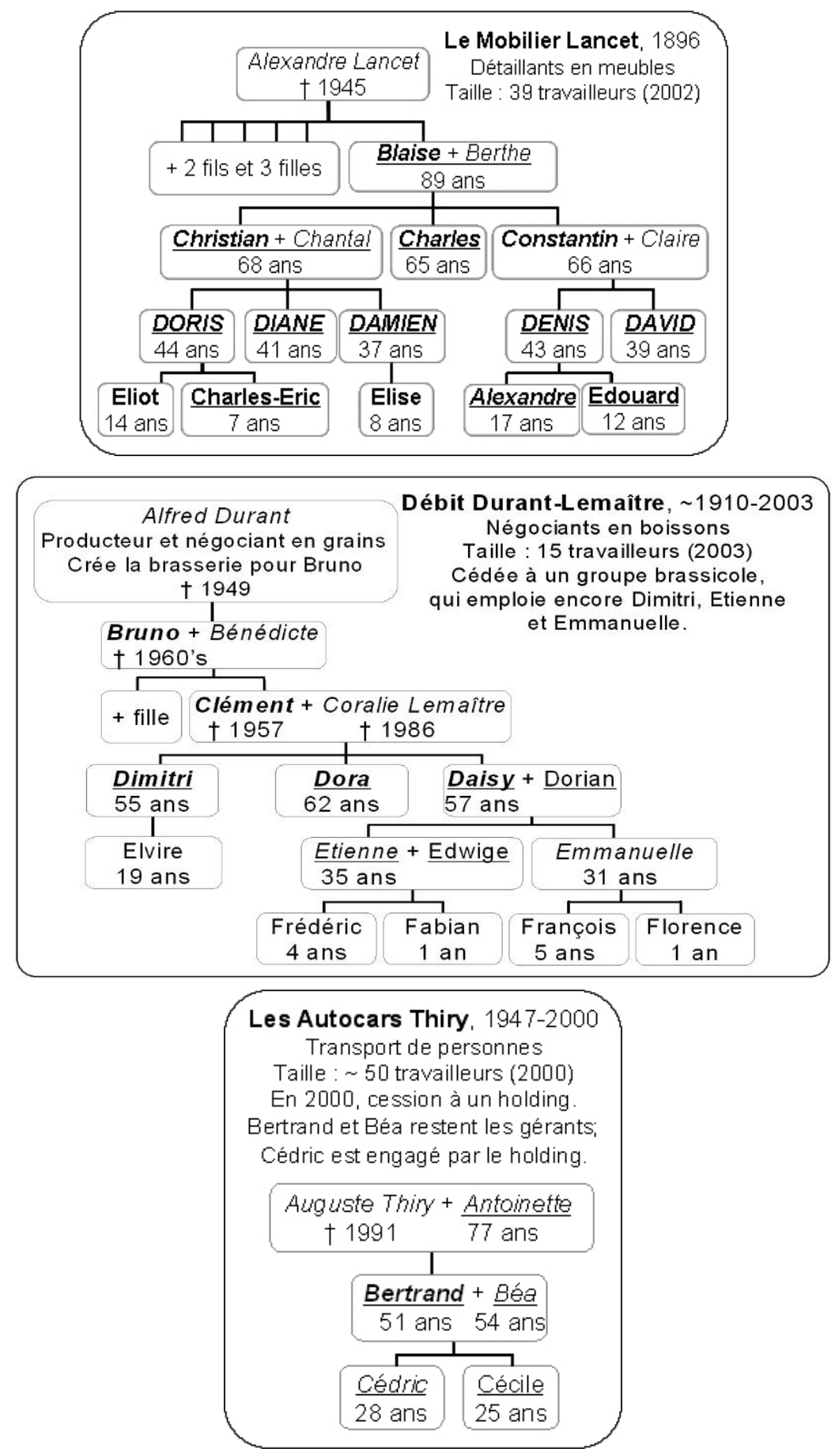
On a abordé chaque entreprise en croisant les entretiens semi-directifs menés auprès de différents membres des familles et de quelques employés. Dans la mesure du possible, les entretiens concernent les dirigeants en place, leurs prédécesseurs, les successeurs potentiels, mais aussi les conjoints et les frères et sœurs qui n'étaient pas ou n'ont pas été directement impliqués dans l'entreprise. Les témoignages sont donc à la fois intra- et intergénérationnels, ils se déplacent au sein de la parentèle afin de faire varier les points de vue et les lectures, de croiser les récits et donc de reconstruire plus objectivement les cheminements, l'histoire de la famille et celle de l'entreprise. Ils donnent aussi accès aux représentations et aux reconstructions qui entourent ces cheminements et révèlent ainsi les dynamiques familiales. Au total, pour ces cinq entreprises, trente-neuf personnes ont été interrogées, âgées de 7 à 89 ans et appartenant jusqu'à quatre générations différentes.

\section{LA LOGIQUE DU DON COMME « PARADIGME »}

Les arbres font bien apparaître la profondeur généalogique des entreprises. D'histoires très longues en histoires plus brèves, aucune de ces entreprises n'est sans passé : toutes ont fait l'objet d'un ou plusieurs transferts, d'un ou plusieurs héritages. Il s'ensuit que chaque dirigeant ou nouvel ex-dirigeant (dans le cas des cessions) est aussi un héritier, et l'on peut poser l'hypothèse que l'entreprise a dû se charger d'un statut symbolique fort au sein de la famille. Elle doit être plus qu'un simple moyen de production et de subsistance, mais un « objet» marqué par celui qui l'a créé puis transmis et par ceux qui l'ont éventuellement transmis à leur tour ; elle doit inspirer une forme de piété filiale, comme on imagine facilement que le fait tel meuble ancien, tel objet précieux ou telle maison qui vient se nouer au fil des générations. De cette hypothèse il découle que leurs dirigeants doivent se sentir dépositaires d'une histoire et usufruitiers de l'entreprise davantage que ses propriétaires, et qu'ils doivent se poser en transmetteurs. Autrement dit, en vertu du statut spécial que l'histoire a donné à l'entreprise, on s'attend à ce qu'existe chez les dirigeantshéritiers une obligation morale de transmission à leurs propres descendants, ou du moins qu'ils soient très favorablement disposés à transmettre l'entreprise au sein de leur famille.

Cette hypothèse de l'héritage comme don intergénérationnel est une relecture de l'Essai sur le don de Mauss. Puisque que sous un aspect libre et gratuit, le don est un cycle de trois moments qui sont autant d'obligations (donner, recevoir, rendre), ayant reçu, il faut rendre ; mais ne pouvant rendre au donateur - donc à ses ascendants -, le donataire rend à ses descendants, qui sont à leur tour appelés à remplir l'obligation du contre-don. Le don est une « roue qui tourne » (Godbout et Charbonneau, 1996) et dans le cas des héritages, elle tourne dans une «circularité rectiligne » (Godbout et Caillé, 1992: 69). La roue tourne sur plusieurs générations et les lie entre elles puisque le don "nourrit ou recrée le social entre les personnes" (ibid. : 32), transmet à la fois du bien et du lien. L'héritage lie ainsi les générations aussi sûrement que sont liés « les anneaux d'une chaîne ", pour reprendre la métaphore de Thomas Mann.

Ne nous y trompons pas, il ne s'agit pas de souscrire à l'animisme qui imprègnerait l'Essai sur le don, cette "sorte de délire fétichiste » (Bazin, 1997: 15), et que lui reprochent nombre de ses commentateurs. Il nous semble que Mauss n'est pas 
dupe de l'animisme du commentaire maori dans lequel il a, dit-il, trouvé « la clef du problème » de l'obligation de rendre (Mauss, 2003 [1923-1924] : 158) :

«Les taonga et toutes propriétés rigoureusement dites personnelles ont un $h a u$, un pouvoir spirituel. Vous m'en donnez un, je le donne à un tiers ; celuici m'en rend un autre, parce qu'il est poussé par le hau de mon cadeau; et moi je suis obligé de vous donner cette chose, parce qu'il faut que je vous rende ce qui est en réalité le produit du hau de votre taonga. » (Ibid. : 159)

Dans la mesure où ce qui est rendu n'est pas nécessairement ce qui a été donné, on comprend que l'esprit, le hau, n'est pas dans la chose mais qu'il est dans la relation entre un donateur et un donataire. Le don est une relation; l'esprit du don peut rester, délié de la chose donnée. Précisément, le geste de donner est un geste de « reconnaissance », un geste créateur de lien : il s'agit « de s'honorer, de se témoigner de l'estime », de " choisir un objet et le tendre vers l'autre comme gage et substitut de soi » (Hénaff, 2002 : 139, 140). En cela, donner, c'est donner un peu de soi, et recevoir, c'est accepter une relation qui n'est pas neutre et dont on ne pourra se délier par simple défection (exit), ainsi qu'on sort d'une relation commerciale pour reprendre la typologie d'Albert Hirschman (1995 [1970] : 32-33). Et le hau n'est que la mise en discours du souvenir de celui qui a donné et de la relation qui s'est fondée (fondue) dans le geste simultané du donner-recevoir. La relation de don est une relation sociale, elle n'est que métaphoriquement une relation d'esprits.

Insistons sur ce point, le parti pris de faire de la transmission des entreprises familiales un don intergénérationnel ne peut se comprendre qu'en se rappelant que les cas exposés dans cet article sont tous des cas dynastiques, c'est-à-dire où les acteurs du moment ne sont pour aucun des fondateurs. Ils ont par conséquent à gérer l'entreprise non seulement en tant que moyen de production soumis, pourrait-on dire, à leur omnipotence de gestionnaire, mais aussi en tant qu'entreprise d'autrui : plane toujours quelque part l'ombre de leurs prédécesseurs. Car, comme le constate Anne Gotman, l'attachement aux biens hérités se manifeste alors même que ceux-ci n'ont pas fait l'objet de prescriptions de la part du défunt (ils n'ont pas été « donnés » à proprement parler) ou que le défunt ne leur était pas particulièrement attaché (1988 : 168). Aussi n'est-il pas question de traiter les biens hérités comme des biens ordinaires. On leur doit un respect proche de celui que l'on devait à la personne qui les a légués et qu'ils personnifient. Ainsi, les héritiers se comportent plus en gardiens et en dépositaires des biens qu'en propriétaires et toute utilisation de l'héritage est soumise à « l'approbation muette » des défunts (Ibid. : 205, 208). C'est comme si un hau habitait les biens légués, l'esprit du parent qui les laisse.

Aussi, si l'on se réfère à cette conception de l'héritage comme don intergénérationnel, on doit s'attendre à ce que les héritiers conçoivent l'entreprise comme un don, la gère avec tout le contenu symbolique, identitaire, que son statut de bien hérité lui confère et qu'ils nourrissent le souhait qu'elle passe à la génération suivante. Or, qu'observe-t-on? Aucun de nos chefs d'entreprise n'est absolument enthousiasmé par l'idée de transmission.

La question finira par se poser de savoir ce que va devenir la société. Vous en discutez déjà?

[Elle réfléchit] Nous, on espère qu'un beau jour y en a un qui arrivera avec une grosse valise et qui nous dira : «On achète le tout. » 
Ça ne vous chagrinerait pas?

Moi, non. Moi, non. Les parents et le grand-père peut-être, peut-être que ça les décevrait, mais moi non. (...) Et puis, disons qu'à partir du moment où mes enfants auraient une situation et qu'ils compteraient sur autre chose que de venir s'installer ici dans un bureau au magasin et attendre que ça tombe, moi j'aimerais autant. (Doris Lancet)

Ou bien, lorsque l'idée de transmission et de continuité plaît à certains dirigeants qui se réfèrent volontiers à leurs ancêtres, elle ne suffit pas à les inscrire dans une démarche active de préparation des héritiers, ni à les persuader que la transmission sera possible, voire qu'elle est souhaitable. Ainsi, si Didier Ribelle évoque spontanément son désir de perpétuation et le présente même comme le moteur de son action, il ne va pas jusqu'à croire que cette perpétuation sera évidente à réaliser :

Mais, l'objectif aussi, c'est quoi ? Tout compte fait nous, on s'en serait peutêtre bien tirés à laisser mourir les choses, mais non, le but c'est d'essayer d'apporter quelque chose aux enfants à un moment donné. C'est la motivation primaire, en tout cas la mienne. Leur dire : «Voilà, on a travaillé toute notre vie, d'abord pour le payer. On a fait quelque chose pour vous, maintenant, ça peut être pour que vous vous impliquiez dedans d'une manière ou d'une autre. » Ils feront peut-être autre chose avec, mais le but c'est d'arriver à ça.

(...)

Pour que les enfants entrent dans la société, qu'est-ce que vous leur conseilleriez comme parcours scolaire ou professionnel?

On aura toujours bien notre petite idée, mais je crois qu'il ne faut pas. Il faut d'abord qu'ils fassent ce qu'ils ont envie de faire. On n'est plus à une époque où on peut dire : «Tu feras ça parce que j'ai besoin de toi pour faire ça », c'est dépassé, c'est tout. Et si les opportunités de la vie font qu'à un moment donné ils ont un intérêt pour, bah on en reparlera. C'est d'abord faire ce dont ils ont envie, ça c'est notre devoir de parents.

Elodie Noiret est, comme Didier Ribelle, animée d'une forte piété dynastique, et cependant, comme lui, elle ne croit pas fermement qu'elle ne sera pas le dernier maillon de la chaîne ni même qu'elle doit tout faire pour ne pas l'être :

Il y a une certaine... une certaine fierté à dire : « Je suis la cinquième génération. » Si je peux encore le transmettre, on verra. J'ai deux petits-enfants qui ont quatre et six ans, c'est un peu tôt... [rires] Mais c'est vrai que c'est une belle histoire qui se continue, on a l'impression de faire partie aussi d'une chaîne quoi, d'être un maillon de la chaîne et que c'est bien. (...)

Vous souhaiteriez que vos enfants reprennent?

Je ne les forcerais pas. Enfin, ça dépend de ce que je vais faire de..., du devenir.

Étant la cinquième génération...

Oui, j'aimerais bien, bien sûr, ce serait une fierté quelque part d'avoir ses enfants qui reprennent, oui, bien sûr. Maintenant, il faut voir comment les choses vont évoluer. Il faut bien être conscient que l'imprimerie est quelque chose qui évolue avec le digital, avec Internet, ça devient très, très différent de ce que ça a été. 
Les cinq cas d'entreprises évoqués ici mettent au jour divers scénarios autour de l'héritage envisagé comme don. Soit, l'entreprise héritée n'a pas pour l'héritier, dans son ressenti, le statut d'un don-héritage qui se déploierait dans un cycle d'obligations verticalisées; soit il y a un ressenti du don, il y a gratitude (Dewitte, 1996) et désir de transmission, mais cette intention de transmission ne se réalise pas pour autant dans une transmission effective ou en attitudes productrices d'héritiers (impliquer les enfants, orienter leurs études, etc.). Face à ces "ratés du contre-don », on peut se demander comment la logique du don influence la disposition à transmettre (l'intention), pourquoi elle ne semble pas s'activer dans tous les cas d'héritage et pourquoi, quand elle est activée, elle ne suffit pas à engendrer un travail de transmission de la part de celui qui désire transmettre.

L'étude de terrain a permis de déceler et d'organiser une série de facteurs qui viennent influencer la dynamique de l'héritage comme don. Certains peuvent être directement mis en rapport avec la logique du don, d'autres relèvent davantage du contexte sociétal global. Sans oublier l'arrière-fond économique dont nul ne peut s'abstraire.

\section{DES ENTRAVES À LA LOGIQUE DU DON}

\section{HÉRITER D'UN BIEN, RECEVOIR LE LIEN?}

Anne Gotman souligne que le cycle du don ne peut s'enclencher comme une mécanique imparable car « entre la transmission et l'héritage se glisse une opération intermédiaire qui consiste (...) à «prendre » et à « recevoir » l'héritage (...). C'est dans cette séquence de la réception et de l'appropriation que l'héritier se construit comme tel. Ses choix seront dictés par des contraintes économiques et identitaires entre lesquelles il sera amené à arbitrer » $(2000: 37)$.

Sans doute faut-il préciser qu'Anne Gotman a surtout étudié des héritages communs (maisons, argent, titres, bijoux, meubles, souvenirs...), mais si de tels héritages ont un potentiel symbolique suffisamment fort pour engager l'identité de l'héritier, on peut raisonnablement penser que les entreprises familiales, parce qu'elles associent un nom et des individus dans une histoire concrète et publique, décuplent ce potentiel lors de leur transmission. Mais pour quelles raisons recevoir est-il identitairement si engageant? Rappelons-le, le don est un «mode de circulation des biens au service du lien social » (Godbout et Caillé, 1992:32). Il est donc à penser comme une relation, comme un rapport social et non comme une série d'actes discontinus et unilatéraux. C'est à ce lien qu'Anne Gotman fait référence quand elle explique que la réception est un moment important du don en ce qu'elle engage l'identité du receveur : accepter le don, le recevoir, c'est accepter le lien, s'affilier au donateur, accepter la filiation. Or, est-il certain que tout héritier a envie de recevoir, que chacun conçoit allègrement d'être repris dans les « rets » des relations de famille (Gotman, $2000: 41$ )?

Quant à l'aspect économique des contraintes qui pèsent sur le moment de l'appropriation de l'héritage, il est ici évident : c'est lui qui donnera le ton à la réception (cadeau ou fardeau ?) et à la volonté de transmettre (est-il sensé d'engager ses enfants dans tel secteur et avec tels instruments ?). Cependant, les contraintes économiques ne l'emportent pas toujours sur les contraintes identitaires, on peut même 
penser que parfois le discours économique soutient, justifie, le mouvement de désaffiliation. C'est ce que l'on constate dans la transmission des Autocars Thiry par Auguste à son fils Bertrand.

En 1975, Auguste subit une thrombose qui l'oblige à arrêter de travailler. Bertrand a 23 ans, il est diplômé en mécanique et travaille depuis cinq ans avec ses parents. Cependant, hors la mécanique, l'activité de ses parents (le transport de personnes) ne le passionne guère : son projet est de lancer une activité de transport routier de marchandises. Mais Bertrand est fils unique, il connaît bien la société et c'est tout naturellement qu'il reprend les rênes de cette entreprise qui fait vivre ses parents, lui-même et quelques ouvriers. Et c'est «tout aussi naturellement» qu'il abandonne le projet de transport routier qui lui tenait à cœur.

L'héritier n'est pas toujours à même de refuser l'héritage ; sur lui s'exercent des pressions familiales, matérielles ou morales qui le contraignent à accepter. «Contraintes ", le mot est dit, voilà une entorse au don, dont il est dit qu'il doit être libre, qu'il ne crée du lien (et donc l'envie de rendre, de maintenir l'attache) que s'il est librement consenti, et librement reçu. Quand il l'évoque, Bertrand ne cache pas le caractère contraint de la reprise, il ne cache pas non plus le peu d'estime qu'il a pour son père, pourtant décédé une dizaine d'années plus tôt, dont il déprécie la personnalité mais aussi la façon de travailler. Deuxième entorse au don, un don n'est don que dès lors qu'il y a reconnaissance, estime entre les parties.

Aucune gratitude (Dewitte, 1996) ne lie Bertrand à son père, aucun sentiment de « dette positive » (Godbout et Charbonneau, $1996: 170)$, c'est à lui-même et à son épouse qu'il attribue le succès de la société qui s'est, il est vrai, fortement développée sous leur direction, et c'est à sa mère qu'il attribue la relative réussite de l'entreprise par le passé. Cette façon de «nier la dette » est une manière de prendre le bien - l'entreprise des parents - sans prendre le lien - l'affiliation au père. Car, en principe, «accepter quelque chose de quelqu'un, c'est accepter quelque chose de son essence spirituelle, de son âme » (Mauss, 2003 [1923-1924] : 161). En insistant bien sur le fait que les Autocars Thiry sont avant tout son œuvre, Bertrand manifeste qu'il ne doit rien à son père. Si on reprenait le vocabulaire animiste des Maori, on dirait que Bertrand a vidé le bien de son hau, du hau de son père. Il a fait du bien hérité son propre bien. Et, en associant son épouse et collaboratrice à la propriété de l'entreprise, il a encore effacé un peu plus la présence du père : habituellement les héritages sont jalousement gardés de l'ingérence des « pièces rapportées » (Gotman, 1988 : 194).

Il n'y a pas de don, il n'y a pas de dette, il n'y a pas d'obligation de contre-don. Avec l'accord de son épouse - et avec beaucoup moins de peine qu'elle, nous ditelle -, Bertrand a vendu l'entreprise familiale à un holding. Il l'a fait cependant que Cédric, leur fils, diplômé en commerce, manifestait l'intention de s'y impliquer, et même ressentait l'obligation morale de le faire, et que quelques aménagements dans la structure financière (ouvrir la propriété à un actionnariat non familial) et organisationnelle de l'entreprise (allonger la ligne hiérarchique, engager du personnel d'encadrement) auraient pu la rendre tout à fait viable entre les mains du fils.

Au-delà du discours de justification économique tenu par Bertrand, son épouse et leur fils, les conditions de la vente, son ambivalence, tendent à démontrer que la cession de l'entreprise continue de réaliser la désaffiliation de Bertrand vis-à-vis de son 
père, et n'est pas un rejet de l'entreprise même ou du métier de chef d'entreprise. En effet, d'une part, Bertrand n'a pas souhaité rester avec son épouse le propriétaire, en quelque sorte, de l'entreprise en devenant, comme les dirigeants du holding le lui proposaient, actionnaire de ce holding par un apport en nature (sa société). D'autre part, en 2003, trois ans après la vente, Bertrand et son épouse, devenus salariés du holding, dirigeaient toujours les Autocars. Sans travailler directement avec ses parents, Cédric était également employé par le holding.

Même si la désaffiliation ne peut expliquer totalement la décision de vendre qu'a prise Bertrand au lieu de transmettre l'entreprise à son fils (sa fille étant, quant à elle, complètement désinvestie), elle participe sans doute à éclairer les modalités de la réception du bien et de sa (non) re-transmission. Comme le remarquent Jacques Godbout et Johanne Charbonneau, les dons sont « le symbole d'une relation de dépendance ». Un moyen de s'en défaire, d'accepter le bien sans accepter le lien est d'« accord[er] au don le sens d'un dû » (1996: 119). Le don est transformé en dû, en droit, qui n'impulse pas un mouvement de circulation; le discours de l'héritier devient : « ceci est à moi, je ne dois rien à personne ».

\section{L'HÉRITIER, RECEVEUR OU CRÉATEUR?}

Dans le premier cas de transmission évoqué, on voit qu'un problème dans la réception de l'héritage vient enrayer le cycle du don. Dans le cas des Meubles Lancet, on ne constate pas, de la part de qui que ce soit, de sentiment négatif directement en rapport avec ce qui a été reçu et avec ceux qui l'ont transmis. Pourtant, les cinq cousins qui représentent la quatrième génération à la tête de l'entreprise évoquent librement la vente du magasin, même si cette vente ne restera qu'une éventualité discrète tant que leurs grands-parents, qu'ils adorent, seront en vie :

\section{Vendre n'est donc pas une idée taboue?}

Non. Non, on en parle. Mais il y a quand même un consensus sur le fait qu'on ne vendra jamais tant que les grands-parents sont en vie. Aucun n'oserait vendre tant qu'ils sont là. Ça les tuerait. Le magasin, c'est leur vie, c'est leur bébé. Les parents seraient sûrement tristes, mais ils nous laisseraient faire. Ça ne les dérangerait pas trop, ils pourraient le vivre assez bien. Mais les grandsparents pas. (Denis Lancet).

Blaise et Berthe, les grands-parents, sont cependant à peu près persuadés que l'entreprise ne restera pas familiale au-delà de la génération en place. Par leur discours, on comprend pourquoi leurs petits-enfants sont moralement autorisés à délaisser l'obligation de conservation et de transmission.

Ça vous a surpris que tous vos petits-enfants viennent au magasin? L'un ou l'autre aurait très bien pu travailler ailleurs.

Berthe : Mais oui, mais comme ils ont toujours travaillé à l'agrandissement de la firme, ça leur tenait quand même à cœur ce qu'ils avaient fait.

Blaise : Ils en deviennent responsables, c'est leur travail, c'est... Vous comprenez? Ça n'a jamais été notre travail à nous, ça a été toujours le travail d'ensemble. Parce que je n'ai jamais considéré mes fils comme mes fils, parce que si on venait au travail à vélo, c'était la main sur l'épaule et, allez, on parlait comme entre amis.

(...) 
C'est une belle fierté, de voir tout ça!

Berthe : Beh oui, on est fiers, on est fiers de ce que... Avec les enfants, hein, parce que c'est pas, à deux on ne l'aurait pas fait, par exemple, si on n'avait pas eu de descendance.

Blaise : Non, parce que tout le monde s'y est mis.

Berthe : Les enfants ont travaillé, ça!

Berthe et Blaise se gardent bien de se poser en seuls bâtisseurs et insistent pour dire que le magasin dans sa forme actuelle est le fruit d'un travail commun à tous. On ne peut dès lors véritablement parler d'héritage et de don puisque l'entreprise est la construction affirmée de trois générations, qui veulent oublier les rapports générationnels, les « horizontaliser », qui œuvrent ensemble, par et pour le groupe familial. Participant à recréer l'entreprise, les héritiers s'approprient l'héritage et il n'est ainsi plus strictement ni donateurs ni donataires. Il y a des associés intergénérationnels, chacun d'eux devient propriétaire et est donc libre de jouir de sa propriété. Ou, pour le dire autrement, le contre-don aux ascendants a été effectué par la fructification du bien attestée par les donateurs, la dette est comme apurée car «la fructification du don reçu est, pour de nombreux parents, le don le plus important de leurs enfants » (Godbout et Charbonneau, $1996: 107$ ).

\section{TRANSMETTRE : DONNER OU EMPOISONNER ?}

Les cousins se savent donc autorisés à se défaire de l'entreprise familiale sans offenser leurs prédécesseurs, dont ils ont, pourrait-on dire, la caution morale ( $c f$. le discours des grands-parents ci-dessus), tout en ayant conscience que cela les affecterait ("Ça les tuerait », nous dit Denis). Ils savent donc que reste dans l'entreprise quelque chose de leurs parents et de leurs grands-parents, qui ne leur appartiendra jamais, la trace de leur passage, les efforts qu'ils y ont mis, le temps qu'ils y ont consacré... Mais la «piété » ne suffit pas à la conservation, la perspective d'une cinquième génération à la tête du magasin est peu souhaitée et semble même peu réaliste :

Ça inquiète que personne ne se profile pour la reprise? Ce serait quand même la cinquième génération...

Ça serait la cinquième, mais... Je pense que maintenant, je ne vais pas dire «on vit au jour le jour avec une société », mais d'année en année. Tout change tellement vite. Il y a de grosses sociétés qui étaient sur le marché depuis des années, on s'était dit que ces gens-là ça fonctionnerait toujours, et puis c'est faillites sur faillites. Nous, on essaie de maintenir la barre, de se remettre en question assez souvent pour que justement on soit toujours compétitifs, maintenant on est surtout basés là-dessus. On n'essaie plus comme nos parents de dire : «Ah ben oui, de toute façon les jeunes sont là. » Non, on les laisse faire ce qu'ils veulent, parce que c'est pas facile. (Diane Lancet)

La transmission d'une entreprise est lourde d'implications pour ses héritiers : il ne s'agit pas ici de recevoir un objet, quelle que soit sa valeur marchande ou symbolique, ou encore d'hériter d'argent, de valeurs mobilières ou immobilières. Nous parlons de PME, d'entreprises toujours sur la brèche et existant justement par cette souplesse à s'adapter aux évolutions des secteurs et des créneaux. Reprendre une société c'est aussi adopter un style de vie, c'est influer sur celui de ses proches, c'est condi- 
tionner sa vie sociale, sa vie conjugale, sa vie parentale... Bref, l'enjeu de la transmission de PME est énorme et il faut une telle foi en la valeur de la position à reproduire, celle de chef d'entreprise avec tout ce que cela implique, pour souhaiter la voir adopter pas ses enfants que des considérations de dettes aux ancêtres et d'obligation morale peuvent paraître bien maigres pour engendrer le désir de transmission et le travail qui doit l'exaucer.

Les parents peuvent, sans que la réception du don ait été problématique, avoir mal vécu leur vie de chef d'entreprise ou la trouver trop difficile pour la souhaiter à leurs enfants ; ou encore, ils peuvent évaluer comme socialement peu désirable la position de chef d'entreprise. Par conséquent, ils élaboreront pour leurs enfants de tout autres projets que la reprise. Ainsi, si le fait de se retrouver à la tête d'une entreprise est perçu comme difficile par le repreneur, on peut comprendre que sa retransmission prenne des allures de « cadeau empoisonné ». C'est le sentiment qui se dégage du constat un peu désabusé de Doris Lancet sur ses conditions de travail :

Vous êtes la quatrième génération, vous accepteriez que vos enfants vous suivent?

Disons que je trouve quand même que c'est lourd. Je trouve que c'est lourd. C'est 9-19, c'est les week-ends, c'est les jours fériés, c'est beaucoup de responsabilités quand même. [soupir] Je ne sais pas. Je ne sais pas. (...) Moi je me dis, si mon aîné peut faire sa place dans un tout autre domaine, pour moi, j'aimerais tout autant. (...) C'est vraiment une... une bagarre tous les jours. Il y a la concurrence, il y a des charges qui sont de plus en plus importantes. On a beaucoup de plaisir à avoir du monde, à avoir un beau magasin, à ce que ça travaille bien et tout, mais c'est beaucoup de travail, c'est beaucoup d'efforts.

Cet aveu d'amertume et cette lecture que fait Doris, tout comme son frère Damien, de sa situation professionnelle, concordent-ils avec le vécu que leurs parents communiquaient de leur propre expérience? Il s'avère que le discours de ces derniers était bien plus teinté d'optimisme que le leur. Propriétaires d'un magasin prospère, vivant confortablement et travaillant avec plaisir dans un secteur florissant, jouissant du prestige public au sein de leur ville, les Lancet de deuxième et troisième générations prolongeaient la belle ascension sociale entamée par Alexandre Lancet. Ils n'avaient aucune raison de souhaiter une autre vie à leurs enfants.

Parfois, les parents eux-mêmes vivent bien leur situation mais ne la perçoivent pas comme ce qu'il y a de mieux pour leurs enfants dans le cadre d'un projet de mobilité sociale - ou de non descension sociale. S'il apparaît que des possibles plus désirables socialement lui sont ouverts, les parents vont donner à leur progéniture des ressources pour les atteindre. Le contre-don à leurs propres prédécesseurs est assuré en amont par leur bonne attitude "fructifiante » de chef d'entreprise (cf. supra) et pourra se poursuivre verticalement, en aval cette fois, en donnant à leurs enfants les ressources pour un meilleur. Le don continue son cycle en quelque sorte en dehors de la matérialité de l'entreprise familiale : on donne mais pas ce que l'on a reçu. L'esprit reste mais s'incarne dans autre chose. C'est aussi ce que François Gresle a constaté, à savoir que les petits patrons peuvent préférer «substituer à la transmission d'un héritage matériel incertain un capital scolaire perçu comme plus essentiel ou plus probant pour l'avenir » (Gresle, 1981: 87). L'entreprise familiale prend alors le statut de «sas », d'étape dans une trajectoire de mobilité (Gresle, 1980 : 2021), non pas de patrimoine à conserver; elle est un moyen, non pas une fin. 
On comprend alors que la transmission soit une question de foi (Champagne, 1986). Dessiner l'avenir de ses enfants autour de l'entreprise familiale implique de croire notamment : au statut du chef d'entreprise (est-ce un statut socialement désirable ? n'y a-t-il rien de mieux qu'on l'on puisse souhaiter pour nos enfants ?), que le mode de vie induit par le statut d'entrepreneur contient un potentiel d'épanouissement personnel pour ses enfants, en la capacité de ses enfants à reprendre l'entreprise, en la situation économique (l'entreprise est-elle viable eu égard à la conjoncture, au marché, aux évolution du contexte industriel, à la structure de l'entreprise ?).

\section{L'OBLIGATION DE LA LIBERTÉ}

On l'a vu au travers du cas de Bertrand Thiry, les dispositions à la transmission sont, notamment, affectées par la contrainte, par la latitude laissée dans l'acceptation de l'héritage. C'est une des règles du don, il y a une obligation de liberté qui fait que la relation n'est pas réductible à l'échange de biens car elle contient toujours une part d'incertitude (celle-là même, précisément, que fuit la transaction économique). On touche ici du doigt un paradoxe : la transmission, qui est une forme de reproduction sociale, exige une certaine liberté pour se réaliser. Une double liberté en fait. D'une part, une liberté subjective, directement liée à la manière dont l'héritier se conçoit au sein du processus de transmission, et, d'autre part, une liberté de délégation qui permet à l'héritier d'agir sur le bien sans trahir l'esprit dont le don procède.

Le premier avatar de cette liberté obligatoire est précisément la liberté qui va agir sur le moral des héritiers - le moral qui est un des principes de la foi évoquée plus haut - et par conséquent sur leurs dispositions à transmettre à leur tour. Daniel Bertaux et Isabelle Bertaux-Wiame ont bien souligné le risque de "réification de l'héritier » inhérent à toute transmission professionnelle, l'inconfort identitaire de l'héritier relégué en quelque sorte au rang d'« objet», de courroie de transmission dans la mécanique de la transmission (1988: 22). Aussi «pour devenir sujet de l'héritage, l'héritier doit agir sur lui en lui imprimant sa propre marque » (ibid.). Mais au-delà de la « marque », l'état de « sujet » de l'héritier dépend également de toute évidence de l'impression qu'il a, ou pas, de pouvoir poser sa marque, de mâ̂triser son destin, d'avoir choisi d'occuper la place qu'il occupe au sein de l'entreprise. La liberté est aussi subjective.

La diversité des parcours et des attitudes des derniers héritiers du Mobilier Lancet par rapport à leur métier nous donne une illustration frappante des effets de la «contrainte de réception» sur les dispositions à transmettre. Sans exclure totalement l'éventualité de la vente, des trois Lancet de quatrième génération à avoir des enfants, Denis est le plus enclin à imaginer l'entrée d'une cinquième génération au magasin. La réserve qu'il émet provient essentiellement d'une incertitude quant aux capacités de gestion de son fils aîné. Il n'émet par contre aucune réserve qui soit liée à la position à reproduire, tant au niveau social qu'au niveau de la qualité de vie (status et situs), ou qui soit liée à la situation économique générale. Damien et Doris, ses cousins qui sont parents eux aussi, adhèrent beaucoup moins à cette idée de continuité familiale.

On peut comprendre ces différences d'attitudes en comparant les chemins qui ont mené chacun à l'entreprise familiale. Abandonnant ses études secondaires, sans diplôme, Doris intègre officiellement l'affaire familiale à 18 ans et met au placard d'autres 
ambitions professionnelles. Conséquence de deux années d'échecs et de déceptions scolaires, ce choix d'entrer au magasin ressemble à un choix en creux, par défaut. La situation de Damien est similaire :

Disons, peut-être que si j'avais réussi mon truc à l'Université [les sciences économiques], peut-être que j'aurais... vogué vers d'autres cieux. Mais, pff, non, là, comme j'ai échoué dans mes études, j'étais bien content de venir ici de toute façon. Mais ça ne me déplaisait pas. Maintenant, je ne sais pas, qu'est-ce qui se serait passé si j'avais eu mon diplôme?

Rejoindre l'entreprise familiale, c'est pour Damien et Doris accéder à un statut que la société - au sens large - ne leur aurait probablement pas permis d'atteindre avec aussi peu de capitaux scolaires : l'entreprise familiale fut une chance de réussir malgré tout, mais c'est aussi un non choix. Doris et Damien ne souhaitent pas que l'entreprise familiale soit pour leurs enfants, comme elle le fut pour eux, une espèce d'heureux pis-aller ; ils leur souhaitent de découvrir d'autres horizons (dont la désirabilité leur paraît plus grande), de faire de véritables choix de vie. Doris résume particulièrement bien le rôle qu'elle accorde à l'entreprise dans le futur de ses fils :

Enfin, en tout cas, je veux leur donner la possibilité de faire autre chose. Je ne veux pas qu'ils viennent ici soi-disant naturellement. S'ils ont envie, s'ils ont une passion, s'il y a vraiment quelque chose de différent qui les attire, je vais tout faire pour qu'ils s'épanouissent dans le domaine qui les intéresse. Je ne veux pas du tout leur imposer de reprendre mon affaire, comme ça, je suis tranquille, j'ai mon repreneur.

Denis ne saurait par contre vivre sa présence au magasin comme la résultante d'un choix par défaut. Il a véritablement choisi de quitter l'école à 16 ans pour entrer au magasin («Rester assis huit heures d'affilée, c'est pas pour moi ») et, loin d'être un abandon, ce fut le moyen de passer en cours du soir un diplôme supérieur en comptabilité. Ce diplôme, un bac+3, fait de Denis une "valeur certifiée » sur le marché du travail, ce que ne sont pas ses cousins. Le magasin de meubles n'a donc pas été pour lui une roue de secours; il n'y a pas d'échec scolaire ou de rêve déçu qui expliquent sa présence dans l'entreprise, ni de découragement qui la grève.

Être indépendant, travailler pour soi, c'est génial (...). Il y a de la liberté, il y a du choix, il y a de l'ouverture, de la variété, on ne fait jamais deux fois la même chose, on rencontre plein de gens. (...) Enfin, si c'était à refaire, il n'y a pas grand-chose que je ne referais pas. J'aime ce que je fais et je ne voudrais pas faire autre chose.

Denis se sent plus que ses deux cousins libre et maître de son destin, son moral et sa foi sont intacts et la transmission peut être envisagée. Face à l'alternative «vendre ou mourir » souvent présentée par les chefs de PME qui s'interrogent sur l'avenir de leur société, Denis répond "grandir », contre ses cousins qui répondent plutôt « vendre ».

Le sentiment d'avoir choisi de recevoir est donc cet avatar subjectif de la liberté obligatoire, essentielle à la dynamique du don. Mais la liberté des héritiers doit aussi être une liberté de délégation : l'héritier doit se sentir libre de transformer le bien, le contenu de l'héritage sans croire trahir l'esprit de ses prédécesseurs qui « habite » le bien transmis. Cette liberté privilégie en quelque sorte l'obligation de résultats contre celle des moyens et assure la possibilité de reconversion du patrimoine et par 
là, bien souvent, sa survie. C'est également une façon pour l'héritier de n'être pas « réifié » par l'héritage puisqu'il peut ainsi «s'approprier comme un élément personnel ce qui en réalité lui a été transmis » (Bertaux et Bertaux-Wiame 1988:2223). Aussi, avant de prononcer l'échec de la transmission, faut-il se demander sur quoi porte le désir de transmission ou le sentiment d'hériter, se demander ce qui « fait héritage ».

Le Débit Durant-Lemaître (négoce de boissons, mise en gérance de cafés) a été vendu en 2003 à un grand groupe brassicole par la quatrième génération, malgré la présence de deux de leurs trois enfants au sein du personnel. L'entreprise est donc sortie du patrimoine familial. Mais cette sortie physique s'est transformée en capitaux financiers aussitôt et intégralement réinvestis dans une société immobilière détenue par la quatrième génération. Si Etienne, l'un des enfants, a souligné la difficulté affective de la séparation d'avec la brasserie, il a largement commenté les activités immobilières de la famille et a évoqué le plaisir pris à accumuler l'argent et à le faire fructifier :

Moi j'aime l'argent pour ce qu'il représente, pas pour ce qu'il est.

Comme un symbole?

Le symbole du travail. Du travail, de ce que mes parents ont fait, de ce que toute la famille a fait. Vous comprenez ce que je veux dire ? C'est pas l'argent pour ce qu'il est, j'en ai rien à cirer que je sois plus riche que l'autre, ça m'est strictement égal. Y en a qui sont plus riches que moi, y en a qui sont plus pauvres, c'est comme ça, et là, là oui, je dirais que je me sens redevable à mes ancêtres, en devoir de faire quelque chose pour que leur patrimoine reste, et éventuellement s'étende. Oui. Oui, je le vois comme ça. Pour moi, c'est une remise à l'échelle de l'évolution de l'espèce. Aller vers quelque chose de meilleur. Progresser. Moi c'est ça mon truc, c'est le progrès.

Ce discours démontre clairement que la non-passation physique d'une entreprise ne signifie pas que le cycle du don s'arrête de tourner ou que l'enchaînement des générations est interrompu. Dans ce cas, parce que les héritiers (ceux en devenir et ceux qui le furent avant eux) se sont donné le droit de transformer l'héritage et ont bénéficié de la nécessaire liberté de délégation, la logique dynastique se réactive autour de nouveaux enjeux de transmission.

\section{DES ENTRAVES SOCIÉTALES À LA LOGIQUE DU DON}

Aux éléments plus directement analysables en tant que liés en propre à la dynamique du don - mais jamais déliés du contexte sociétal et économique qui les baigne -, il faut ajouter ceux qui concernent cette fois la société en général et qui ne peuvent pas ne pas affecter la dynamique de la transmission intergénérationnelle.

\section{LE SOI DANS LA FAMILLE}

Comme on l'a vu, l'intention de transmission ne fait pas l'unanimité, mais elle n'est pas non plus tout à fait absente des projections des chefs d'entreprise. Cependant quand on pose la question des moyens, de ce qu'eux, en tant que parents et chefs d'entreprise, font ou pensent qu'ils devraient faire pour donner vie à ces projections, la réponse est qu' «il ne faut pas forcer » et qu' "il faut laisser faire aux 
enfants ce dont ils ont envie ». Pourquoi les parents désireux de transmettre ne travailleraient-ils pas à la construction de leurs successeurs, par exemple, en exhortant leurs enfants à se former dans des domaines utiles à l'entreprise, en les employant dans l'entreprise pendant week-ends et vacances, en leur présentant l'entreprise comme un futur à fortement envisager?

Une telle proposition est parfaitement en décalage avec l'« hédonisme individualiste » (Kellerhals et Montandon, 1991 : 30), avec la norme contemporaine d'épanouissement de soi. C'est pourtant un devenir imposé qu'ont vécu nombre d'interviewés et nombre de leurs prédécesseurs qui ne sont pas si âgés. Leur a-t-on demandé, par exemple, s'ils voulaient étudier qui la chimie (Carl Ribelle), qui la menuiserie (Christian Lancet), qui la comptabilité (Désiré Noiret) ou si la mécanique des camions ne les passionnait pas davantage que le transport par cars (Bertrand Thiry) ? Bien sûr, ces injonctions parentales n'ont pas forcément été vécues sur le mode de la contrainte : parce qu'un travail d'inculcation efficace fait que le devoir devient vouloir (Pinçon et Pinçon-Charlot, 1999 : 92) et que l'horizon des possibles pouvait se borner objectivement (et pas seulement subjectivement) à la proposition parentale, ces injonctions ont rarement été ressenties comme telles.

Autre temps, autres mœurs : sociologues et philosophes s'accordent à définir la norme d'épanouissement de soi comme la norme dominant aujourd'hui nos sociétés, du moins dans les classes moyennes (de Singly, 1996; Taylor, 2002). En accord avec cette norme, la famille aurait changé de fonction : d'une fonction de transmission d'un patrimoine (tangible et intangible), sa fonction première serait devenue la révélation de soi. C'est la «fonction de Pygmalion » de la famille (de Singly, 1996). La relation familiale est ainsi mise au service de l'individu et non plus au service du groupe. C'est en effet ce discours de l'épanouissement personnel que les parents et certains grands-parents tiennent lorsqu'on leur demande de s'exprimer sur l'avenir de leurs éventuels successeurs, et cela qu'ils soient largement favorables à un prolongement familial de l'entreprise ou plus réticents à cet égard ( $C$ 'est d'abord faire ce dont ils ont envie, ça c'est notre devoir de parents », disait Didier Ribelle).

La société ne permet donc plus d'imposer un futur à ses enfants, en tout cas pas ouvertement car ce serait par trop dévier des valeurs dominantes, et la « matière à travailler » ne s'en laisserait sans doute pas conter. Le travail de reproduction, si cette reproduction est désirée, doit passer par des voies plus cachées, des actions plus sourdes ; mais même discret, il s'accompagne toujours d'une forme de violence et d'une prise de contrôle sur l'individu qui sont à présent honnies. Aussi la norme d'accomplissement de soi et la fonction de Pygmalion dévoluent aux parents ne peuvent-elles compromettre la naissance même du désir de transmission ? N'en devientil pas un désir coupable, inavouable?

\section{LA CONCURRENCE DES SOCIALISATIONS}

La particularité des entrepreneurs, comme celle des agriculteurs et d'autres professions indépendantes, tient dans ce que, à la différence des salariés, ils possèdent leurs propres moyens de production. Ces moyens de production forment un patrimoine dont la transmission exige de disposer de repreneurs capables de s'en servir. À charge des parents de produire ces repreneurs, d'éduquer, de socialiser leurs en- 
fants en ce sens, même si, comme on vient de le voir, quand il est trop directif ce travail est en désaccord avec la norme de l'épanouissement de soi.

Dans une enquête sur les causes de la diminution des successions agricoles, Patrick Champagne relève que les agriculteurs vivaient autrefois dans une espèce d'autarcie sociale par laquelle « la famille paysanne réussissait à contrôler étroitement tous ses membres aussi longtemps que rien ne venait la concurrencer sérieusement. Elle parvenait à envelopper totalement et continûment la vie des enfants (...) et à leur imposer ainsi ses valeurs, son mode de vie, sa définition de l'excellence professionnelle, bref, sa vision du monde et de l'avenir » (1986:46). Bien sûr, la situation des entrepreneurs n'a jamais rien eu de commun avec l'ancien isolement des paysans, mais l'idée que pour qu'un jeune ait envie de reprendre l'exploitation « il ne faudrait pas qu'il ait goûté à autre chose » (Ibid. : 47) fait tout à fait sens. Or, aujourd'hui, «l'éducation est simultanément menée par plusieurs agents [et] quand on étudie les pratiques éducatives familiales, [il faut] analyser (...) les influences éducatives des autres instances de socialisation » (Kellerhals et Montandon ; 1991 : 9). Les enfants ont en effet plus que jamais l'occasion de «goûter à autre chose » ou, plus justement, ils savent que d'autres goûts existent. Beaucoup plus que ce qu'ont connu leurs aînés.

Il y a une perte de contrôle de la famille sur la socialisation que divers éléments contribuent à expliquer; le sujet est complexe, on ne fera que l'effleurer. L'explication que donne Denis Lancet au fait que tous les cousins de la quatrième génération soient venus travailler au magasin dépeint en quelques traits cette ouverture de la socialisation :

\begin{abstract}
Avant, il n'y avait pas de loisirs pour les enfants comme il y en a maintenant. On allait à l'école avec mon frère, puis on faisait nos devoirs et quand on avait fini on s'ennuyait. (...) Alors on descendait au magasin qui était juste en dessous de l'appartement. A 14 ans, on vendait déjà des meubles et les clients aimaient bien. On allait aux foires aussi avec nos parents et on ne demandait pas mieux. Mes gamins, je leur demande parfois s'ils veulent m' accompagner, mais ça ne les intéresse pas. A l'époque on n'avait pas tant de possibilités de loisirs, d'études, ni de liberté que maintenant. (...) Nous, quand on s'ennuyait, on allait au magasin ; mon fils, s'il s'ennuie, il va voir des copains, il va jouer à la Playstation, il va regarder la télé. Et puis, il a plus de liberté. Les parents disaient ce qu'il fallait faire et puis c'est tout. Si j'avais voulu faire autre chose, mon père ne m'en aurait pas empêché, mais est-ce qu'on entrevoyait même la possibilité de faire autre chose ? Je ne crois pas. Pour nous cinq, ça c'est un peu passé comme ça, par la force des choses.
\end{abstract}

On retrouve dans cette réponse la fameuse norme d'épanouissement qui fait que les parents d'aujourd'hui laissent à leurs enfants plus de liberté qu'eux-mêmes n'en ont eu, ce qui n'est pas sans liens avec l'offre de loisirs dont la liberté permet de profiter (et que les parents sont devenus comme interdits d'interdire). Mais le plus grand facteur de détournement est sans nul doute l'allongement et la diversification de la scolarité. La certification scolaire donne une prise sur les devenirs professionnels des individus puisqu'elle constitue, idéalement, le sésame pour un emploi hors marché du travail familial, marché auquel est à peu près condamné celui dont les compétences ne sont pas labellisées par quelque mention officielle. Quant à la diversité de l'offre de formation, elle ouvre l'horizon des futurs, subjectivement (savoir 
qu'il est d'autres possibles) et objectivement (y accéder). A cet égard, on se souviendra que le parcours scolaire des cousins Lancet n'est probablement pas pour rien dans leur entrée au magasin. C'est aussi une observation qui se fait dans les milieux du petit patronat, que les parents ont tendance à se positionner " contre trop d'école » (mais « un peu quand même ») pour éviter que les horizons ne s'ouvrent trop largement par-delà l'entreprise familiale (Amiot, 1991 : 96-110).

Un autre «phénomène de société » participe à diluer le contrôle parental sur la socialisation. D'une façon générale, on admettra qu'une socialisation à l'entrepreneuriat comme stratégie de reproduction, que cette stratégie réponde ou pas à une visée consciente (Bourdieu, 1989 : 386-406), aura d'autant plus de chance d'être efficace qu'il y aura accord des deux parents sur son contenu. Si un tel accord n'est sans doute pas évident à trouver dans les couples unis (Zarca, 1993 : 283), que fautil imaginer de la congruence des projets parentaux quand, en cas de divorce, le couple parental survit mal au couple conjugal (de Singly, 1996:122)? A cela s'ajoutent les effets de l'organisation des droits de garde : selon le parent qui bénéficie des plus larges plages, l'enfant peut se trouver très peu en contact avec l'entreprise, tant en contact physique avec elle qu'en contact avec l'ethos du parent qui y est impliqué. Notre corpus de cas ne nous permet cependant que de supposer les conséquences des séparations parentales : aucun des héritiers n'est un «enfant du divorce » et les enfants des divorcés sont encore trop jeunes pour que l'on puisse observer l'épaisseur du lien qui les attache à l'entreprise.

On voit que les évolutions sociétales complexes, simplifiées ici autour des axes génériques de l'épanouissement personnel et de la perte de contrôle sur la socialisation, mettent à mal l'évidence des transmissions «naturelles », qui «vont de soi », antienne pourtant chantée par la plupart des interviewés quand il s'agit d'expliquer leur entrée dans la société familiale. Ces chefs d'entreprise, parents de jeunes enfants ou d'adolescents, qui sont acquis, ainsi que leurs discours le dénotent et parfois leurs pratiques (par exemple, inscrire les enfants à un maximum d'activités culturelles et sportives), aux normes dominantes et qui sont conscients du peu de contrôle qu'elles leur laissent exercer sur leurs enfants, ces parents peuvent-ils ardemment émettre le vœu d'une transmission? Et lorsqu'ils caressent ce vœu, peuvent-ils suffisamment croire que cette transmission est réalisable pour s'engager dans l'action?

\section{CONCLUSION}

Sur le plan théorique, la re-transmission des entreprises familiales pensée dans les termes du don maussien a toutes les séductions d'un phénomène complexe qui serait dompté, réduit à la mécanique bien huilée d'un système d'obligations. Sur le plan empirique, on voit que la logique du don est facilement enrayée. Les conditions pour qu'elle s'active et s'assortisse d'effets sont très difficiles à rassembler. Cette logique ne peut en tout état de cause suffire à baliser la complexité des contextes et des enjeux de la transmission des entreprises familiales, qui sont des systèmes qui relèvent de la sphère familiale, domestique, privée - sphère privilégiée du don (Godbout et Caillé, 1992 : 37), mais qui brassent également des éléments débordant suffisamment cette sphère pour relativiser les obligations propres au système de don.

A un niveau macroscopique, la transmission des entreprises familiales ne peut en effet se délier du contexte sociétal qui impose ses valeurs, ses normes, qui propose 
des configurations familiales nouvelles et des potentiels de cheminements différents dans l'espace social (on pense ici à la démocratisation scolaire) ; elle n'est pas non plus indépendante, tant s'en faut, des recompositions économiques et des péremptions industrielles. Macro-freins donc, mais micro-freins aussi : à l'intérieur même de la sphère privée, l'histoire personnelle, les effets de la relation aux parents, les parcours de vie (la reprise de l'entreprise comme contrainte, choix ou pis-aller), le vécu de la situation, les projets de mobilité conçus pour ses propres enfants... sont autant d'éléments qui tiennent à une «microsociologie familiale » et qui ne sauraient faire des héritages un écoulement tranquille de flux matériels et symboliques.

Pourtant, heuristiquement, il est fécond de faire «comme si » la logique du don fonctionnait par défaut dans les retransmissions d'entreprise et «comme si » elle était capable de soumettre à sa loi toutes les contingences. Ainsi, si le terrain nous a bien montré que dette aux aïeux et volonté d'un apurement lignager sont des figures trop rhétoriques du mythe, on a également pu constater que des considérations de piété aux ascendants, de gratitude, de respect du bien transmis existent dans la plupart des cas. Ces considérations prouvent qu'avec le bien se transmet du lien, elles prouvent que la transmission est performatrice de relations, crocheteuse de mailles entre les générations et que l'attitude par rapport à la transmission est aussi, précisément, une manière de resserrer ou de filer les mailles. Toute mythologie a son fondement de réalité, celle de l'héritage pas moins qu'une autre.

La logique du don doit être prise pour ce qu'elle est, ni plus ni moins, un « échafaudage » dirait Goffman (1973 [1959] : 240), une « loi sociale » dirait Weber, qui apporte non pas « une connaissance de la réalité sociale, mais [qui est] seulement un des multiples moyens que la pensée utilise à cet effet » (Weber, 1992 [1904] : 159). Penser l'héritage multigénérationnel comme don et faire du don un paradigme dans l'étude des re-transmissions permet en effet de déceler et d'organiser ce qui lui « fait échec ». En cela, même s'il est empiriquement défaillant, le "paradigme du don » s'avère un outil précieux de préhension et d'ordonnancement du social, et, par là même, de sa compréhension.

Delphine LOBET

Aspirante FNRS

Université libre de Bruxelles Institut de Sociologie

Centre de Sociologie des Organisations avenue Jeanne 44 - CP124 1050 BRUXELLES delphine.lobet@ulb.ac.be $+32(0) 2 / 650.34 .81$ 
Aмiot M. 1991. Les misères du patronat... Le monde des Petites et Moyennes Entreprises Industrielles et de leurs patrons, Paris, L'Harmattan, coll. Logiques sociales.

Bauer M. 1992 [1990]. Pas de sociologie d'entreprise sans sociologie de ses dirigeants, in R. Sainsaulieu (dir.), L'entreprise, une affaire de société, Paris, Presses de la Fondation Nationale des Sciences Politiques, coll. Références, p. 148174.

BAZIN J. 1997. La chose donnée, in Critique, tome 53, n 596-597, p. 7-24.

BERTAUX D., BERTAUX-WIAME I. 1988. Le patrimoine et sa lignée : transmissions et mobilité sociale sur cinq générations, Life stories/Récits de vie, $\mathrm{n}^{\circ} 4, \mathrm{p} .8-25$.

Boltanski L., Chiapello E. 1999. Le nouvel esprit du capitalisme, Paris, Gallimard, NRF Essais.

BouRdiEU P. 1989. La noblesse d'État. Grandes Écoles et esprit de corps, Paris, Minuit, coll. Le sens commun.

Champagne P. 1986. La reproduction de l'identité, in Actes de la recherche en sciences sociales, $\mathrm{n}^{\circ} 65$, p. 41-64.

DewitTE J. 1996. Il ne fallait pas. Notes sur le don, la dette et la gratitude, Revue du Mauss, $\mathrm{n}^{\circ}$ 8, p. 102-113.

Godbout J. T., CAILlE A. 1992. L'esprit du don, Paris, La Découverte, coll. Textes à l'appui.

Godbout J.T., Charbonneau J. (avec LemieuX V.) 1996. La circulation $d u$ don dans la parenté. Une roue qui tourne, Montréal, INRS-Urbanisation.

GodBout J. T. 2000. Le don, la dette et l'identité. Homo donator vs homo aecuménicus, Paris, La Découverte, Bibliothèque du Mauss.

Godbout J. T. 2004. L'actualité de l'«Essai sur le don », Sociologie et sociétés, vol. $36, \mathrm{n}^{\circ} 2$, p. $177-188$.

GOFFMAN E. 1973 [1959]. La mise en scène de la vie quotidienne. 1. La présentation de soi, Paris, Minuit.

Gotman A. 1988. Hériter, Paris, PUF, coll. Économie en liberté.

Gotman A. 1995. Dilapidation et prodigalité, Paris, Nathan, coll. Essais et recherches.

Gotman A. 2000. Héritage, dilapidation et construction identitaire, in Héritages. Les enjeux psychiques de la transmission, Actes des IV èmes Journées d'études de l'Association des Psychologues de Franche-Comté, novembre 1999, p. 33-44, Paris, L'Harmattan, coll. Psychanalyse et civilisations.

GRESLE F. 1980. Indépendants et petits patrons. Pérennité et transformations d'une classe sociale, Thèse présentée devant l'Université de Paris V le 8 juin 1978, Lille, Atelier de Reproduction des Thèses.

GRESLE F. 1981. L'univers de la boutique. Famille et métier chez les petits patrons $d u$ Nord (1920-1975), Lille, Presses Universitaires de Lille.

HÉNAFF M. 2002. De la philosophie à l'anthropologie. Comment interpréter le don ?, Esprit, février 2002, p. 135-158.

HiRsChMAN A. O. 1995 [1970]. Défection et prise de parole, Paris, Fayard, coll. L'espace du politique.

HugRON P. 1998. La régie d'entreprises familiales, in Revue internationale de Gestion, vol. 23, p. 37-40. 
Kellerhals J., Montandon C. 1991. Les stratégies éducatives des familles, Lausanne, Delachaux et Niestle, coll. Actualités pédagogiques et psychologiques.

MANN T. 1965 [1901]. Les Buddenbrook. Le déclin d'une famille (trad. G. Bianquis), Paris, Fayard, coll. Horizon libre.

MAUSS M. 2003 [1923-1924]. Essai sur le don. Forme et raison de l'échange dans les sociétés archaïques, Sociologie et anthropologie, p. 143-279, Paris, PUF, coll. Quadrige.

PINÇON M., PINÇON-CHARLOT M. 1999. Nouveaux patrons, nouvelles dynasties, Paris, Calmann-Lévy.

SINGLY F. de. 1996. Le soi, le couple et la famille, Paris, Nathan, coll. Essais et recherches.

TAYLOR C. 2002. Le malaise de la modernité, Paris, Cerf, coll. Humanités.

WEBER F. 2001. Pour penser la parenté contemporaine, in D. Debordeaux, P. Strobel (dir.), Les solidarités familiales en questions, Paris, Maison des Sciences de l'Homme, coll. Droit et société, Série sociologie, p. 73-106.

WEBER M. 1992 [1904]. L'objectivité de la connaissance dans les sciences et la politique sociale, in Essais sur la théorie de la science, Paris, Plon, Pocket, coll. Agora.

ZARCA B. 1993. L'héritage de l'indépendance professionnelle selon les lignées, le sexe et le rang dans la fratrie, in Population, $\mathrm{n}^{\circ} 2$, p. 275-306. 